UvA-DARE (Digital Academic Repository)

\title{
Statistical properties of SGR $1806-20$ bursts
}

Göğüș, E.; Woods, P.M.; Kouveliotou, C.; van Paradijs, J.; Briggs, M.S.; Duncan, R.C.; Thompson, C.

Published in:

Astrophysical Journal Letters

DOI:

$10.1086 / 312583$

Link to publication

Citation for published version (APA):

Göğüș, E., Woods, P. M., Kouveliotou, C., van Paradijs, J., Briggs, M. S., Duncan, R. C., \& Thompson, C. (2000). Statistical properties of SGR 1806-20 bursts. Astrophysical Journal Letters, 532(2), L121-L124. https://doi.org/10.1086/312583

\section{General rights}

It is not permitted to download or to forward/distribute the text or part of it without the consent of the author(s) and/or copyright holder(s), other than for strictly personal, individual use, unless the work is under an open content license (like Creative Commons).

\section{Disclaimer/Complaints regulations}

If you believe that digital publication of certain material infringes any of your rights or (privacy) interests, please let the Library know, stating your reasons. In case of a legitimate complaint, the Library will make the material inaccessible and/or remove it from the website. Please Ask the Library: https://uba.uva.nl/en/contact, or a letter to: Library of the University of Amsterdam, Secretariat, Singel 425, 1012 WP Amsterdam, The Netherlands. You will be contacted as soon as possible. 
The Astrophysical Journal, 532:L121-L124, 2000 April 1

(C) 2000. The American Astronomical Society. All rights reserved. Printed in U.S.A.

\author{
STATISTICAL PROPERTIES OF SGR 1806-20 BURSTS \\ Ersin GöĞÜş, ${ }^{1,2}$ Peter M. Woods, ${ }^{1,2}$ Chryssa Kouveliotou, ${ }^{2,3}$ Jan van Paradius, ${ }^{1,4}$ \\ Michael S. Briggs, ${ }^{1,2}$ Robert C. Duncan, ${ }^{5}$ and Christopher Thompson ${ }^{6}$ \\ Received 1999 December 21; accepted 1999 February 7; published 2000 March 7
}

\begin{abstract}
We present statistics of SGR 1806-20 bursts, combining 290 events detected with the Rossi X-Ray Timing Explorer/Proportional Counter Array, 111 events detected with the Burst and Transient Source Experiment, and 134 events detected with the International Cometary Explorer. We find that the fluence distribution of bursts observed with each instrument are well described by power laws with indices $1.43,1.76$, and 1.67, respectively. The distribution of time intervals between successive bursts from SGR 1806-20 is described by a lognormal function with a peak at $103 \mathrm{~s}$. There is no correlation between the burst intensity and either the waiting times until the next burst or the time elapsed since the previous burst. In all these statistical properties, SGR 1806-20 bursts resemble a self-organized critical system, similar to earthquakes and solar flares. Our results thus support the hypothesis that the energy source for soft gamma repeater bursts is crustquakes due to the evolving, strong magnetic field of the neutron star, rather than any accretion or nuclear power.
\end{abstract}

Subject headings: gamma rays: bursts — stars: individual (SGR 1806-20) — X-rays: bursts

\section{INTRODUCTION}

Soft gamma repeaters (SGRs) are a rare class of objects characterized by their repetitive emission of low-energy gamma-ray bursts. SGR bursts last $\sim 0.1 \mathrm{~s}$, and their spectra are usually well described by an optically thin thermal bremsstrahlung (OTTB) model with $k T \sim 20-40 \mathrm{keV}$. Three of the four known SGRs are associated with slowly rotating $\left(P_{\text {spin }} \sim\right.$ 5-8 s; Mazets et al. 1979; Kouveliotou et al. 1998; Hurley et al. 1999), ultrastrongly magnetized $\left(B \gtrsim 10^{14} \mathrm{G}\right.$; Kouveliotou et al. 1998, 1999a) neutron stars positioned within or near young supernova remnants. For a review of the burst and persistent emission properties of SGRs, see Kouveliotou (1999b) and Hurley (2000).

Cheng et al. (1996) reported similarities between particular statistical properties of a sample of 111 SGR 1806-20 bursts (observed with the International Cometary Explorer [ICE] between 1979 and 1984) and earthquakes. They noted that the distribution of the event energies of both phenomena follow a power law $d N \propto E^{-\gamma} d E$ with index $\gamma \sim 1$.6. Furthermore, they found that the cumulative waiting times between successive SGR bursts and earthquakes are similar. Laros et al. (1987) noted that the distribution of waiting times between the ICE SGR 1806-20 bursts follow a lognormal function, which was also seen between microglitches of the Vela pulsar (Hurley et al. 1994). Using the same data set, Palmer (1999) showed that, similar to earthquakes, some SGR 1806-20 bursts may originate from relaxation systems. Gögüus et al. (1999) studied a set of 1024 bursts from SGR 1900+14; 187 bursts were detected with the Burst and Transient Source Experiment (BATSE) aboard the Compton Gamma-Ray Observatory $(C G R O)$ and 837 bursts were detected with the Proportional

\footnotetext{
${ }^{1}$ Department of Physics, University of Alabama in Huntsville, Huntsville, AL 35899.

${ }^{2}$ NASA Marshall Space Flight Center, SD-50, Huntsville, AL 35812.

${ }^{3}$ Universities Space Research Association.

${ }^{4}$ Astronomical Institute "Anton Pannekoek," University of Amsterdam, 403 Kruislaan, 1098 SJ Amsterdam, Netherlands.

${ }^{5}$ Department of Astronomy, University of Texas, RLM 15.308, Austin, TX $78712-1083$

${ }^{6}$ Department of Physics and Astronomy, University of North Carolina, Philips Hall, Chapel Hill, NC 27599-3255.
}

Counter Array (PCA) on the Rossi X-Ray Timing Explorer (RXTE) during an active period of the source in 1998. We found that their energy distribution is consistent over 4 orders of magnitude with a power law of index $\gamma=1.66$. The distribution of waiting times between successive bursts also follows a lognormal function, which peaks at $\sim 49 \mathrm{~s}$. We discussed the idea that SGRs, like earthquakes and solar flares, are manifestations of self-organized critical systems (Bak, Tang, \& Wiesenfeld 1988). All of these results are consistent with the idea that SGR bursts are caused by starquakes, which are the result of a fracture of the crust of a magnetically powered neutron star, or "magnetar" (Duncan \& Thompson 1992; Thompson \& Duncan 1995, 1996).

SGR 1806-20 exhibited sporadic bursting activity from the launch of BATSE (in 1991 April) until 1996 October (Kouveliotou et al. 1993) when it entered a burst active phase emitting 38 bursts over the following 2 months. In 1996 November, a series of pointed observations were performed with the RXTE/ PCA over a period of 2 weeks. These observations led to the discovery of 7.47 s pulsations from SGR 1806-20 and confirmed its nature as a magnetar (Kouveliotou et al. 1998). In these 2 weeks, RXTE/PCA recorded a total of 290 bursts. $^{7}$ In the BATSE data, SGR 1806-20 burst activity was persistent but variable from 1996 October up to 1999 October with a total of 116 recorded bursts. In this Letter, we present a comprehensive study of the statistical properties of SGR 1806-20 by combining several databases. Sections 2,3 , and 4 describe the CGRO/BATSE, RXTE/PCA, and ICE observations, respectively. Our results are presented in $\S 5$ and discussed in $\S 6$.

\section{BATSE OBSERVATIONS}

In our analysis we have used DISCriminator Large Area detector (DISCLA) data with coarse energy resolution (four channels covering energies from $25 \mathrm{keV}$ to $\sim 2 \mathrm{MeV}$ ), spectroscopy time-tagged event (STTE) data, and spectroscopy high energy resolution burst (SHERB) data with fine energy binning (256 channels covering energies from $15 \mathrm{keV}$ to $\sim 10 \mathrm{MeV}$ )

\footnotetext{
${ }^{7}$ Examples of RXTE/PCA observations of SGR 1806-20 can be seen at http://gammaray.msfc.nasa.gov/batse/sgr/sgr1806/.
} 


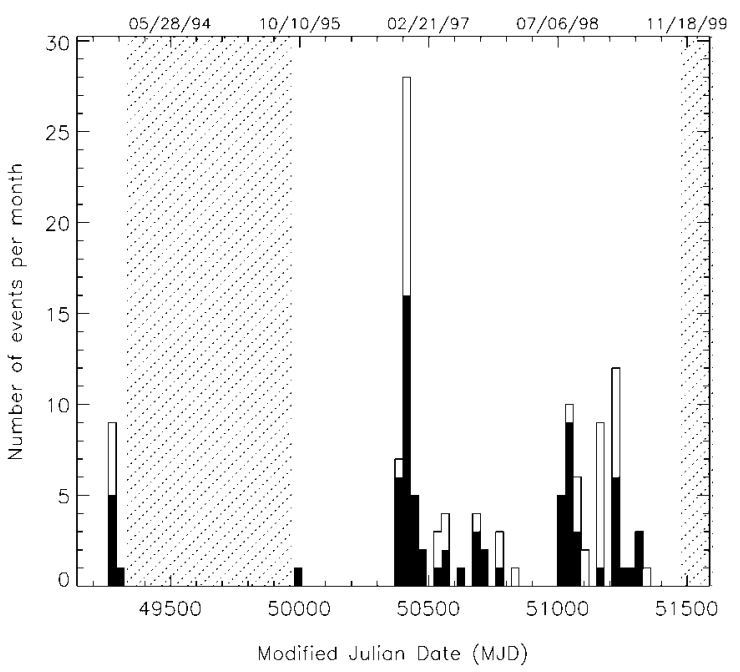

FIG. 1.-Plot of activity history of SGR $1806-20$ as seen with BATSE. Shaded regions denote the time intervals within which the off-line untriggered burst search was not performed. The filled portions inticate the number of events within each time bin that led to an on-board trigger.

from the spectroscopy detectors. A detailed description of BATSE instrumentation and data types can be found in Fishman et al. (1989).

BATSE triggered on 74 bursts between 1993 September and 1999 June. For 32 of the brightest events, STTE or SHERB data with detailed spectral information were obtained. The background subtracted spectra were fit to OTTB and powerlaw models. The OTTB model, $F(E) \propto E^{-1} \exp (-E / k T)$, provided suitable fits $\left(0.76<\chi_{\nu}^{2}<1.36\right)$ to all spectra, with temperatures ranging between 18 and $43 \mathrm{keV}$. The power-law model failed to fit most of the spectra. The weighted mean of the OTTB temperatures for this sample of 32 events is $20.8 \pm 0.2 \mathrm{keV}$.

To increase our burst sample, we performed an off-line search for untriggered BATSE events from SGR 1806-20 using a method explained in detail by Woods et al. (1999a). Figure 1 shows the overall BATSE burst activity history of SGR 1806-20. We limited our search during active phases of the source. We found, in addition to the 74 triggered events, 42 untriggered bursts during the time intervals 1993 September 13-November 20 and 1995 September 7-1999 October 26. Of these 116 events, 111 events (triggered and untriggered) had DISCLA data and were sufficiently intense to allow spectral fitting. Because of the long DISCLA data integration time (1.024 s) compared to typical SGR burst durations ( 0.1 s), we could estimate only the fluence for each event. We fit the background-subtracted source spectrum to an OTTB model with a fixed $k T$ of $20.8 \mathrm{keV}$, a reasonable choice considering the fairly narrow $k T$ distribution of the triggered bursts derived above. We find that the burst fluences range between $1.4 \times$ $10^{-8}$ and $4.3 \times 10^{-6} \mathrm{ergs} \mathrm{cm}^{-2}$. For a distance to SGR $1806-20$ of $14.5 \mathrm{kpc}$ (Corbel et al. 1997) and assuming isotropic emission, the corresponding energy range is $3.5 \times$ $10^{38}-1.1 \times 10^{41}$ ergs. In comparison, the energies of SGR $1900+14$ bursts seen with BATSE range between $1.1 \times 10^{38}$ and $1.5 \times 10^{41} \mathrm{ergs}$ (Göğüş et al. 1999) and those of SGR 1627-41 between $8.0 \times 10^{37}$ and $5.5 \times 10^{41}$ ergs (Woods et al. 1999b).

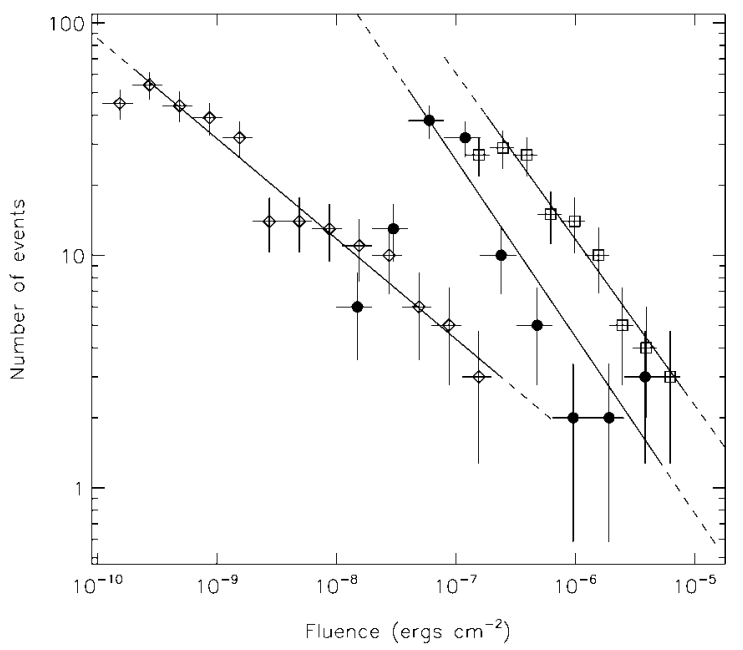

FIG. 2.-Differential fluence distributions of SGR 1806-20 bursts as seen by RXTE (diamonds), BATSE (circles) and ICE (squares). The lines are obtained fitting a power-law model with the maximum likelihood technique. The solid lines show the intervals used in the fit, and the dashed lines are the extrapolations of each model.

\section{RXTE OBSERVATIONS}

We performed 13 pointed observations of SGR 1806-20 with the RXTE/PCA, for a total effective exposure time of $\sim 141$ ks between 1996 November 5 and 18. We searched PCA standard 1 data $(2-60 \mathrm{keV})$ with 0.125 time resolution for bursts using the following procedure. For a given $0.125 \mathrm{~s}$ time bin at time $t$, we estimated a background count rate by fitting a firstorder polynomial to $5 \mathrm{~s}$ of data before (from $t-8$ to $t-3 \mathrm{~s}$ ) and after (from $t+3$ to $t+8 \mathrm{~s}$ ) the bin searched. Bins with count rates exceeding 125 counts per $0.125 \mathrm{~s}$ were assumed to include burst emission and were excluded from the background intervals. A burst was defined as any continuous set of bins with count rates above $5.5 \sigma$ of the estimated background. For the typical PCA count rate of $12-18$ counts per $0.125 \mathrm{~s}$ in this energy band, the $5.5 \sigma$ level corresponds to $\sim 20-25$ counts in a $0.125 \mathrm{~s}$ bin. We found 290 events and measured the count fluence of each burst by simply integrating the backgroundsubtracted counts over the bins covering the event.

To compare the integrated count fluences obtained with the PCA to the BATSE fluences, we determined a conversion factor between the two as follows. First, we searched for bursts observed with both instruments and found eight such events (five of which had triggered BATSE). Assuming a constant OTTB model as described in $\S 2$, we estimated the fluence of these bursts. We then computed the ratio of the BATSE fluence to the PCA counts of each common event. These ratios fall within a fairly narrow range $\left(3.5 \times 10^{-12}\right.$ and $8.1 \times 10^{-12} \mathrm{ergs} \mathrm{cm}^{-2}$ counts ${ }^{-1}$ ). Their weighted mean is $5.5 \times 10^{-12} \mathrm{ergs} \mathrm{cm}^{-2}$ counts $^{-1}$ with a standard deviation $\sigma=1.3 \times 10^{-12} \mathrm{ergs} \mathrm{cm}^{-2}$ counts $^{-1}$. The mean is very close to the one estimated for SGR $1900+14$ (Göğüs et al. 1999) and consistent with the idea that SGR bursts have a similar spectral shape. Using this conversion factor, we find that the fluences of the PCA bursts from the PCA range from $1.2 \times 10^{-10}$ to $1.9 \times 10^{-7} \mathrm{ergs} \mathrm{cm}^{-2}$ and the burst energies range from $3.0 \times 10^{36}$ to $4.9 \times 10^{39} \mathrm{ergs}$.

\section{ICE OBSERVATIONS}

From 1978 to 1986, the Los Alamos gamma-ray burst detector on board the ICE satellite (Anderson et al. 1978) almost 


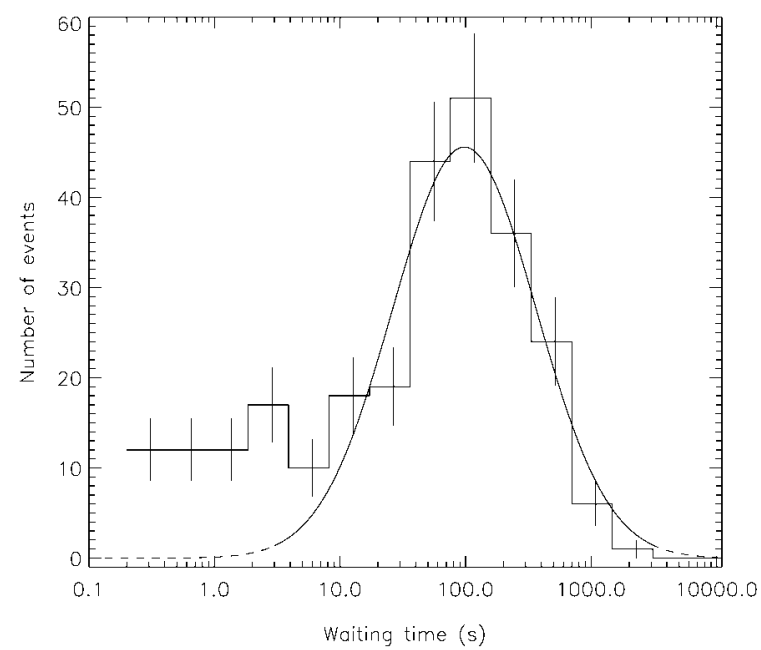

FIG. 3.- Histogram of the waiting times $\Delta T$ between successive $R X T E / \mathrm{PCA}$ bursts from SGR 1806-20. The line shows the best-fit lognormal function. The solid portion of the line indicates the data used in the fit. The excess of short intervals above the model is due to the double peaked events as explained in the text.

continuously observed the Galactic center region within which SGR $1806-20$ is located. It detected 134 bursts from the source between 1979 January 7 and 1984 June 8 (Laros et al. 1987, 1990; Ulmer et al. 1993). Combining observational details given by Ulmer et al. (1993) and energy spectral information obtained by OTTB fits to bursts (at energies $E>30 \mathrm{keV}$ ) given by Fenimore, Laros, \& Ulmer (1994) and Atteia et al. (1987), we estimate that the ICE burst fluences range from $1.5 \times$ $10^{-8}$ to $6.5 \times 10^{-6} \mathrm{ergs} \mathrm{cm}^{-2}$ and their corresponding isotropic energies are between $3.6 \times 10^{38}$ and $1.6 \times 10^{41}$ ergs.

\section{STATISTICAL DATA ANALYSIS AND RESULTS}

From the previous three sections, we clearly see that the BATSE and ICE detection sensitivities are quite similar, with PCA extending the $\log N-\log P$ distribution to lower values. We now combine all databases to a common set, enabling several statistical analyses.

1. Burst fluence distributions. - To eliminate systematic effects due to low count statistics or binning, we have employed the maximum likelihood technique to fit the unbinned burst fluences. A power law fit to 92 BATSE fluences between $5.0 \times 10^{-8}$ and $4.3 \times 10^{-6} \mathrm{ergs} \mathrm{cm}^{-2}$ yields a power-law exponent, $\gamma=1.76 \pm 0.17$ (68\% confidence level). Bursts with fluences below $5.0 \times 10^{-8} \mathrm{ergs}^{-2}$ were excluded to avoid undersampling effects due to lower detection efficiency. Figure 2 shows the BATSE fluences binned into equally spaced logarithmic fluence steps (circles). Similarly, we fit the 266 PCA fluence values between $1.7 \times 10^{-10}$ and $1.9 \times 10^{-7} \mathrm{ergs}$ $\mathrm{cm}^{-2}$ to a power-law model and obtain a best-fit exponent value of $1.43 \pm 0.06$ (see Fig. 2; diamonds for PCA). Finally, the 113 ICE fluences between $1.8 \times 10^{-7}$ and $6.5 \times 10^{-6}$ ergs $\mathrm{cm}^{-2}$ yield $\gamma=1.67 \pm 0.15$ (see Fig. 2; squares for ICE). We find that the power-law indices obtained for BATSE and ICE agree well with each other, while the index obtained from PCA is marginally lower.

We fit the ICE fluences to a power law times exponential model and to a broken power-law model to search for evidence of a turnover claimed by Cheng et al. (1996). Neither model provides a statistically significant improvement over a single

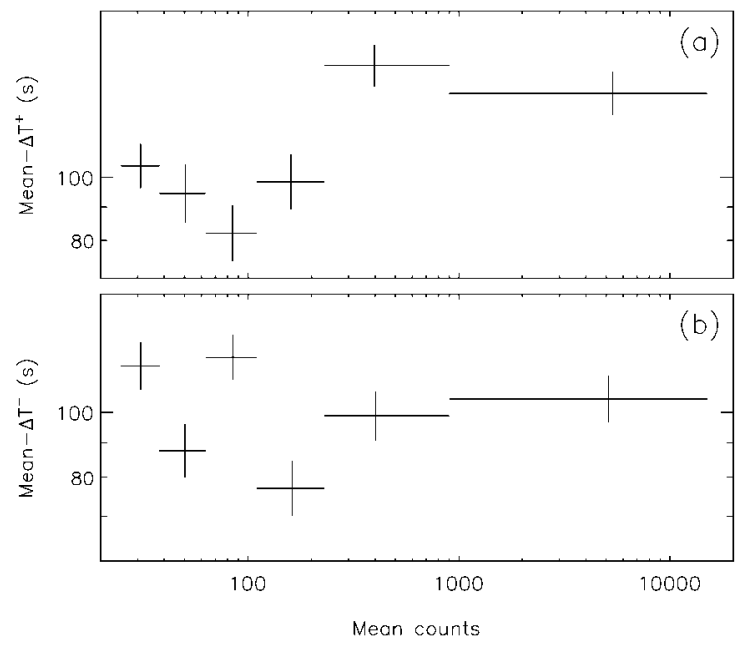

FIG. 4.-(a) Plot of lognormal mean waiting times till the next burst $\left(\Delta T^{+}\right)$vs. mean total counts. No correlation is seen $(\rho=-0.2, P=0.70)$. (b) The plot of lognormal mean elapsed times since the previous burst $\left(\Delta T^{-}\right)$vs. mean counts does not show any correlation either $(\rho=0.4, P=$ $0.46)$.

power-law fit. It is important to note that there is no evidence of a high-energy cutoff or a break in the energy distribution (see Fig 2).

2. Waiting times distribution.-To measure the waiting times between successive SGR 1806-20 bursts, we identified 22 RXTE observation windows containing two or more bursts without any gaps. We then determined 262 recurrence interval times $\Delta T$ (i.e., time difference between successive bursts). Figure 3 shows a histogram of the $\Delta T$ 's, which range from 0.25 to $1655 \mathrm{~s}$. We have fit the $(\Delta T)$-distribution to a lognormal function and found a peak at $\sim 97 \mathrm{~s}$ (with $\sigma \sim 3.6$ ). This fit does not include waiting times less than $3 \mathrm{~s}$ to avoid contribution of double-peaked events in which the second peak appears shortly $(\sim 0.25-3 \mathrm{~s})$ after the first one. To correct for biases due to the RXTE observation window ( 3000 s), we performed extensive numerical simulations and found that the intrinsic peak of the distribution should be at $\sim 103 \mathrm{~s}$. Note that observation windows with no bursts may represent a longwaiting-time tail which is additional to the lognormal distribution.

To investigate the relation between the waiting time till the next burst $\left(\Delta T^{+}\right)$and the intensity of each burst, we divided the 290 events sample into six intensity intervals, each of which contains approximately 50 events. We fit the $\Delta T^{+}$-distribution also to a lognormal distribution and determined each peak mean $\Delta T^{+}$(which range from 82 to $148 \mathrm{~s}$ ) and the mean counts for each of the six groups. We show in Figure $4 a$ that there is no correlation between $\Delta T^{+}$and the total burst counts (the Spearman rank-order correlation coefficient $\rho=-0.2$ with a probability that this correlation occurs in a random data set of $P=0.70$ ). Similarly, we investigated the relation between the elapsed times since the previous burst $\left(\Delta T^{-}\right)$and the intensity of the bursts. We find that mean $\Delta T^{-}$extends from 77 to $120 \mathrm{~s}$. Figure $4 b$ shows that there is also no correlation between mean $\Delta T^{-}$and the burst counts $(\rho=0.4, P=0.46)$.

\section{DISCUSSION}

The fluence distributions of the SGR 1806-20 bursts seen with ICE and BATSE are well described by single power laws 
with indices $1.67 \pm 0.15$ and $1.76 \pm 0.17$, respectively, while RXTE bursts have an index of $1.43 \pm 0.06$. These indices are similar to those found for SGR 1900+14 (1.66; Gögüüs et al. 1999) and SGR 1627-41 (1.62; Woods et al. 1999b). The ICE and BATSE values are consistent with one another, over nearly the same energy range but at different epochs. This suggests that SGR event fluence distributions may not vary greatly in time; therefore, we combine the ICE and BATSE values to calculate a "high-energy" index, $\gamma=1.71 \pm 0.11$. The difference between the "low-energy" (RXTE) index and the highenergy index is insignificant $(\sim 2.3 \sigma)$; more high-energy data are needed to determine whether there is a break in the distribution.

Power-law energy distributions have also been found for earthquakes with $\gamma=1.4-1.8$ (Gutenberg \& Richter 1956; Chen, Bak, \& Obukhov 1991; Lay \& Wallace 1995, p. 376) and solar flares, $\gamma=1.53-1.73$ (Crosby et al. 1993; Lu et al. 1993). This is a typical behavior seen in self-organized critical systems. The concept of self-organized criticality (Bak et al. 1988) states that subsystems self-organize owing to some driving force to a critical state at which a slight perturbation can cause a chain reaction of any size within the system. SGR power-law fluence distributions, along with a lognormal waiting time distribution, support the idea that systems responsible for SGR bursts are in a state of self-organized criticality. We believe that in SGRs, the critical systems are neutron star crusts strained by evolving magnetic stresses (see Thompson \& Duncan 1995).

Cheng et al. (1996) suggested that there is a high-energy cutoff in the cumulative energy distribution of SGR 1806-20 bursts seen by ICE. In a cumulative energy distribution, the values of neighboring points are correlated; consequently, judging the significance of apparent deviations is very difficult. For these reasons we used a maximum likelihood fitting technique and displayed the differential energy distributions (e.g., Fig. 2). We find no evidence for a high-energy cutoff in the ICE data of SGR $1806-20$ up to burst energies $\sim 10^{41}$ ergs. It should be noted, however, that a high-energy cutoff or turnover must exist because otherwise the total energy diverges.

The distribution of waiting times of SGR 1806-20 bursts observed with RXTE is well described by a lognormal function, similar to that found by Hurley et al. (1994) for the bursts seen with ICE. The waiting times of the RXTE events are on average shorter than the ones observed with $I C E$, maybe due to different burst active phase of the source or to instrumental sensitivity (the PCA is more sensitive to weaker bursts than ICE, and the system displayed plenty of weaker bursts as well as strong ones in 1996), or a combination of both. Recently Gögüss et al. (1999) showed that the recurrence time distribution of SGR 1900+14 bursts observed with RXTE is also a lognormal function which peaks at $\sim 49 \mathrm{~s}$. The lack of any correlation between the intensity and the waiting time until the next burst agrees well with the results of ICE observations of SGR 1806-20 (Laros et al. 1987). This behavior, also seen in SGR 1900+14 (Göğüş et al. 1999) confirms that the physical mechanism responsible for SGR bursts is different from systems in which accretionpowered outbursts take place (e.g., the rapid burster [Lewin et al. 1976] and the bursting pulsar [Kouveliotou et al. 1996]).

The burst activity of SGR $1806-20$ over the last 3 years is considerably different from that of SGR $1900+14$. After a long period with almost no bursts, BATSE recorded 200 bursts from SGR 1900+14 between 1998 May and 1999 January, with remarkably low activity thereafter. On the other hand, after SGR 1806-20 reactivated in 1996, it continued bursting on a lower rate, with 18 bursts in 1997, 32 in 1998, and 18 in 1999 through October. The latest RXTE observations of SGR 1806-20 in 1999 August revealed that smaller scale bursts are still occurring occasionally in this system, whereas contemporaneous RXTE observations of SGR 1900+14 do not show burst activity of any size. This continuation of burst activity may prevent the deposition of very large amounts of stress in the crust. Therefore, in SGR 1806-20 it may be less likely to expect, in the near future, a giant flare from this source, as the ones seen on 1979 March 5 from SGR 0526-66 (Mazets et al. 1979) and on 1998 August 27 from SGR 1900+14 (Hurley et al. 1999).

We are grateful to the referee, D. Palmer, for his very constructive comments. We acknowledge support from NASA grant NAG5-3674 (E. G., J. v. P.), the cooperative agreement NCC 8-65 (P. M. W.), NASA grants NAG5-7787 and NAG57849 (C. K.), Texas Advanced Research Project grant ARP028, and NASA grant NAG5-8381 (R. C. D.).

\section{REFERENCES}

Anderson, K. A., et al. 1978, IEEE Trans. Geosci. Electron., GE-16, 157 Atteia, J.-L., et al. 1987, ApJ, 320, L105

Bak, P., Tang, C., \& Wiesenfeld, K. 1988, Phys. Rev. A, 38, 364

Chen, K., Bak, P., \& Obukhov, S. P. 1991, Phys. Rev. A, 43, 625

Cheng, B., et al. 1996, Nature, 382, 518

Corbel, S., et al. 1997, ApJ, 478, 624

Crosby, N. B., et al. 1993, Sol. Phys., 143, 275

Duncan, R. C., \& Thompson, C. 1992, ApJ, 392, L9

Fenimore, E. E., Laros, J. G., \& Ulmer, A. 1994, ApJ, 432, 742

Fishman, G. J., et al. 1989, in Compton Observatory Science Workshop, ed. W. N. Johnson (NASA Conf. Publ.; Washington, D.C.: NASA), 3 Göğüus, E., et al. 1999, ApJ, 526, L93

Gutenberg, B., \& Richter, C. F. 1956, Bull. Seism. Soc. Am., 46, 105

Hurley, K., et al. 1999, Nature, 397, 41

- 2000, in AIP Proc., 5th Huntsville Symp. on Gamma-Ray Bursts, ed.

R. M. Kippen, R. S. Mallozzi, \& G. J. Fishman (New York: AIP), in press Hurley, K. J., et al. 1994, A\&A, 288, L49

Kouveliotou, C. 1999b, Proc. Natl. Acad. Sci., 96, 5351
Kouveliotou, C., et al. 1993, Nature, 362, 728

. 1996, Nature, 379, 799

1998, Nature, 393, 235

1999a, ApJ, 510, L115

Laros, J. G., et al. 1987, ApJ, 320, L111

1990, Proc. 21st Int. Cosmic-Ray Conf. (Adelaide), 1, 68

Lay, T., \& Wallace, T. 1995, Modern Global Seismology (San Diego: Academic)

Lewin, W. H. G., et al. 1976, ApJ, 207, L95

Lu, E. T., et al. 1993, ApJ, 412, 841

Mazets, E. P., et al. 1979, Nature, 282, 587

Palmer, D. M. 1999, ApJ, 512, L113

Thompson, C., \& Duncan, R. C. 1995, MNRAS, 275, 255 . 1996, ApJ, 473, 322

Ulmer, A., et al. 1993, ApJ, 418, 395

Woods, P., et al. 1999a, ApJ, 524, L55 1999b, ApJ, 519, L139 